\title{
Percepción de violencia y sexismo en estudiantes universitarios
}

\author{
Perception of violence and sexism in university students
}

\section{ISSN 2071-8748 \\ E-ISSN 2218-3345 \\ (c) (1) $\$$ \\ BY NC SA}

URI: http://hdl.handle.net/11298/877

DOI: http://dx.doi.org/10.5377/entorno.v0i66.6726

\author{
Roxana Yolanda Castillo-Acobo \\ Docente e investigadora principal \\ Afiliada a la Universidad Nacional de \\ San Agustín de Arequipa \\ rcastilloa@unsa.edu.pe1 \\ ORCID: 000-0002-6467-7796
}

\author{
Sandra Choqque-Soto \\ Coinvestigadora \\ Afiliada a la Universidad Nacional de \\ San Agustín de Arequipa \\ schoqque@unsa.edu.pe2 \\ ORCID: 0000-0001-8247-9315
}

Recibido: 30/07/2018 Aprobado: 12/12/18

\section{Resumen}

El estudio indaga sobre las relaciones de pareja de los estudiantes universitarios, por cuanto muchas veces los estudiantes que no participan con regularidad en su formación académica, indican sentirse afectados por relaciones afectivas que les causan desmotivación, depresión, cambios de alimentación, estados emocionales alterados, dependencia emocional. El objetivo es analizar las percepciones de los estudiantes en torno a la violencia, sexismo desde una perspectiva de género que crean sujeción o subordinación en las relaciones de pareja. Participaron 250 estudiantes, 115 hombres y 110 mujeres, elegidos de forma aleatoria simple de las 47 escuelas profesionales áreas de biomédicas, ingenierías y sociales; se aplicó la escala estandarizada sobre

\section{Abstract}

The study delves about the couple's relationships of university students, since many times students who do not participate regularly in their academic formation point out they feel affected by affective relationships that cause them lack of motivation, depression, changes in their eating habits, altered emotional states and emotional dependence. The objective is to analyze the perceptions of students about violence and sexism from a gender perspective, which creates attachment or subordination in the couple's relationships. Participants included 250 students, 15 men y 110 women, chosen at random from the 47 professional schools in biomedical, engineering and social areas; the standardized scale on Affectivity and Sexism of the Instituto Andaluz de la Mujer

1 Dra. Roxana Yolanda Castillo Acobo, docente e investigadora principal de la Escuela de Trabajo Social de la Universidad Nacional de San Agustín de Arequipa (Unsa). Directora del Observatorio de Género y Calidad de Vida y del Instituto de Investigaciones Sociales, de la Unsa. rcastilloa@unsa.edu.pe

2 Mg. Sandra Choqque Soto, docente de la Escuela Profesional de Trabajo Social de la Unsa, integrante del Observatorio de Género y Calidad de Vida-Unsa. schoqque@unsa.edu.pe. 
Afectividad y Sexismo del Instituto Andaluz de la Mujer (2001). Los resultados según escala indican que los y las estudiantes "están de acuerdo y tienen" información de las desigualdades existentes entre hombres, mujeres y que la violencia sexista afecta a las mujeres. Tipifican a los hombres como agresivos, más violentos. Reconocen que las mujeres al callar y aguantar situaciones de violencia las expone a violencia de género, acoso sexual u otros. Sin embargo, siendo los códigos de cotejo muy sutiles éstos crean percepciones que claramente tipifican al sexismo benévolo y paternalista, además de ser resultado de la socialización diferencial de género, hace a las mujeres más emocionales.

\section{Palabras clave}

Violencia en la educación; Violencia - Investigaciones; Violencia contra la mujer; Delitos contra la mujer.
(Andalusian Institute for Woman (2001)) was applied. The scale results indicate that the students "agree and have" information about inequalities between men and women, and that gender violence affects women. They typify men as aggressive, more violent. They recognize that women, by remaining silent and enduring situations of violence, are exposed to gender violence, sexual harassment or others. However, since the codes of comparison are very subtle, they create perceptions that clearly typify sexism as benevolent and paternalistic, as well as being the result of differential gender socialization which makes women more emotional.

\section{Keywords}

Violence in education; Violence - Investigations; Violence against women; Crimes against women.

\section{Introducción}

Las situaciones de violencia sexual, expresiones de subordinación, debilidad, actitudes de desprotección de las mujeres en las relaciones afectivas y en el entorno inmediato pueden ser indicios de acoso y violencia sexual en las cuales los estudiantes universitarios están involucrados. La realidad de estos está mediada por las condiciones de las carreras, por visiones y actitudes sexistas que marcan sus relaciones interpersonales entre las parejas. La universidad es un espacio donde también se dan hechos de violencia. Así la vida afectiva es importe en el desarrollo emocional y personal de los estudiantes al presentarse efectos negativos que repercuten en el fluido desempeño académico, en la motivación, atención, en los conflictos entre pares estudiantiles, y lo más preocupante, el estar expuestos a riegos de violencia ante las parejas. En Perú, Vara-Horna \& López-Odar (2016), realizaron un estudio con muestra de 3.500 mujeres y 3.458 hombres, estudiantes universitarios, en 34 universidades del país, se identificó que hay un $65 \%$ de estudiantes que fueron agredidas por su pareja o expareja; que prevalece la violencia psicológica, en $62 \%$; la violencia física, en $29,4 \%$; con los efectos académicos de mayor impacto en los hombres por el menor rendimiento académico, $55 \%$, y por deseos de deserción, $57 \%$, señalando que se pierden 20.4 días de clase por cada estudiante agredida y agresor.

Rottenbacher (2010), a escala Perú, hace comparaciones sobre sexismo. Por áreas de estudio, se tiene que existen diferencias significativas en el sexismo benévolo paternalista, de modo que los estudiantes de ingenierías presentan puntajes mayores que los de sociales. $Y$ en función de la universidad de procedencia, se encontró que los estudiantes de la universidad pública tenían mayores niveles de sexismo hostil, benévolo paternalista $y$ ambivalente que quienes estudian en una universidad privada, es decir, que el sexismo está mediado por la condición socioeconómica de las personas; sugiere que los varones tienen más prejuicios que las mujeres, y que sus ideas son más sexistas, atribuyendo ciertos papeles a la mujer. En ese sentido, los varones tienen, además de mayores niveles de sexismo hostil, puntuaciones más altas en sexismo benévolo heterosexual, y en el sexismo benévolo paternalista, lo que quiere decir que, mientras hay varones que asumen un papel de protectores de la mujer, hay otros que asumen uno más violento. Estos resultados son similares a los de Cárdenas, Lay, González, Calderón 
y Alegría (2010), quienes encontraron que los estudiantes universitarios varones de Chile tienen puntajes más altos en sexismo hostil y benevolente que las estudiantes. Otros estudios han reportado que el sexismo se encuentra significativamente relacionado con la expresión de ira y hostilidad (Garaigordobil, 2015), lo que supone que el sexismo hostil es un factor de riesgo de incurrir en conductas francamente violentas para quienes obtuvieron puntajes más altos en la dimensión de sexismo hostil.

Estos hechos se presentan en la universidad, sin embargo, no se cuenta con datos precisos lo que motiva el estudio de las relaciones entre el sexismo y la violencia con la interrogante, ¿cuáles son las percepciones que tienen los estudiantes en torno a la violencia, el sexismo y los papeles de género, ¿qué crea subordinación, dominación y vulnerabilidad en sus relaciones de pareja? El objetivo es analizar las percepciones de los estudiantes en torno a la violencia y el sexismo desde una perspectiva de género, que crean sujeción o subordinación en las relaciones de pareja. Los objetivos específicos son: 1) identificar la información y el conocimiento sobre violencia de los estudiantes universitarios, 2) precisar las razones o causas de la violencia en pareja en los estudiantes universitarios, 3) identificar la valoración del amor y las relaciones de pareja y 4) conocer las estrategias de afectividad en las relaciones de pareja de los estudiantes universitarios.

Tratándose de una universidad pública, cuyo financiamiento depende del tesoro público, que acoge a estudiantes de clases media y media baja, se dificulta la movilidad o ascenso social mediante la profesionalización, sumándose a ello situaciones de abandono estudios universitarios por violencia, tanto en caso del agresor, como en persona agredida. Además, la entidad pública aporta capital profesional-humano al desarrollo de la sociedad; de allí que dichas experiencias de los estudiantes deben ser estudiadas y comprendidas humanamente; todo lo cual crea la función de disminuir contextos de violencia que puedan afectar a la sociedad en todos sus niveles, así como a la persona misma en el futuro mediato y en el largo plazo.

\section{Metodología}

La investigación es de corte transversal, de nivel comparativo cuantitativo. La finalidad es medir en estudiantes de las diferentes áreas académicas, hombres y mujeres, la escala de afectividad y el sexismo. Participaron 250 estudiantes, (115 hombres y 110 mujeres), elegidos de forma aleatoria simple de las 47 escuelas profesionales, áreas de biomédicas, ingenierías y sociales. Se aplica una escala para medir la percepción de los estudiantes sobre las variables de violencia y sexismo en las relaciones de pareja; se selecciona el instrumento estandarizado sobre afectividad y sexismo del Instituto Andaluz de la Mujer en 2001, midió las siguientes áreas: información y conocimiento sobre violencia, razones o causas de violencia en pareja, la valoración del amor y las relaciones de pareja, estrategias de afectividad en las relaciones de pareja, siendo una escala útil para identificar las percepciones sobre afectividad en las relaciones. La escala tiene cuatro valoraciones (del 4 al 1), respecto de la cual los estudiantes libremente seleccionan las opciones que perciben se dan con mayor frecuencia en su relación o en la relación de parejas de sus compañeros estudiantes. La puntuación se marca así: 4 TD = Totalmente de acuerdo, 3 DA = De acuerdo, 2 ED = En desacuerdo y 1 TD $=$ Totalmente en desacuerdo.

El tiempo de aplicación promedio fue de 30 minutos, por cuanto se inicia con etapa de información y se busca el consentimiento del participante. Se tuvo en cuenta aplicar el instrumento solo a la persona que aceptó, asegurando la confidencialidad de la información, lo cual es necesario para tener respuestas sinceras y reales. El procesamiento de datos se realizó importándolos de Excel mediante SPSS; se mantiene la valoración de escala del 4 al 1 y no porcentajes, por cuanto la muestra no permite hacer inferencias y menos generalizaciones de los resultados a toda la población universitaria.

\section{Resultados}

El proceso de acopio de datos fue con instrumento estandarizado sobre sexismo del Instituto Andaluz de la Mujer (2001), el cual consta de ocho componentes: información y conocimiento, causas de la violencia en parejas, percepción estrategias enamorados o noviazgo, amor y relaciones de pareja, 'el amor significa encontrar a', 'por amor sería capaz de', 'si tu pareja te daña o hace sufrir' y 'la frase que más te gustaría escuchar de tu pareja'. Participaron 250 estudiantes (115 hombres y 110 mujeres) de entre los 18 y 23 años de edad, de las tres áreas académicas: ingenierías, biomédicas y sociales. El método de elección fue aleatorio simple. Si bien hay algunas carreras profesionales que mantienen estereotipos de género, ese no fue el criterio de inclusión. La finalidad es seleccionar tanto a hombres como a mujeres en relación con las variables del estudio y contar con la opinión de ambos géneros sobre sus relaciones afectivas. 


\section{Información y conocimiento sobre violencia de los estudiantes universitarios}

\section{Gráfico 1. Información y conocimiento}

\begin{tabular}{|c|l|}
\hline $\begin{array}{c}\text { Ítem } \\
1\end{array}$ & $\begin{array}{l}\text { Cuando una mujer maltrata a un hombre también es } \\
\text { violencia, pero no de género. }\end{array}$ \\
\hline 2 & $\begin{array}{l}\text { En nuestro país hay todavía desigualdades importantes } \\
\text { entre hombres y mujeres. }\end{array}$ \\
\hline 3 & $\begin{array}{l}\text { Los casos de maltrato suelen aparecer ya desde el } \\
\text { principio, de novios. }\end{array}$ \\
\hline 4 & $\begin{array}{l}\text { Solo puede hablarse de maltrato cuando a la mujer el } \\
\text { hombre le pega. }\end{array}$ \\
\hline 5 & $\begin{array}{l}\text { Los hombres maltratadores suelen ser violentos dentro y } \\
\text { fuera de casa. }\end{array}$ \\
\hline 6 & $\begin{array}{l}\text { Si la mujer no aguantara realmente la situación de maltrato, } \\
\text { se iría de la casa. }\end{array}$ \\
\hline
\end{tabular}

Los estudiantes tienen información sobre las desigualdades entre hombres y mujeres, y acerca de que la violencia sexista afecta a las mujeres a nivel de sociedad y en ámbito universitario; asimismo, tipifican las características de los hombres como agresivas, más violentas. Reconocen que las mujeres, al callar y aguantar situaciones de violencia, que presenta riesgos. Es decir, que las situaciones actuales de violencia, sexismo, feminicidio y acoso sexual son

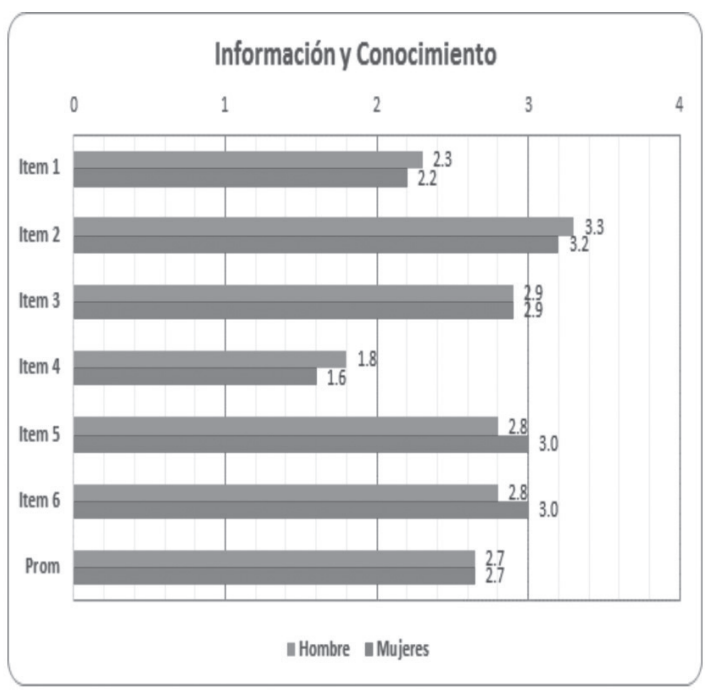

conocidos, detectados por los estudiantes en general. Las percepciones, también en general, coinciden en que hombres y mujeres, con puntuaciones de 3.3, están de acuerdo con los ítems 2.5 y 6 . Están de acuerdo con que "si la mujer no aguantará realmente la situación de maltrato, se iría de la casa", es decir, que de manera implícita perciben que hay una dependencia emocional de las mujeres en las relaciones de pareja, lo que crea notoria vulnerabilidad.

\section{Razones o causas de violencia en pareja en los estudiantes universitario}

\section{Gráfico 2. Causas de violencia en parejas}

\begin{tabular}{|c|l|}
\hline $\begin{array}{c}\text { Ítem } \\
1\end{array}$ & Pérdida de nervios del hombre \\
\hline 2 & Machismo e ideas machistas \\
\hline 3 & Trastornos mentales del maltratador \\
\hline 4 & Abusos sufridos por maltratadores \\
\hline 5 & Consumo de alcohol y otras drogas \\
\hline 6 & Problemas que preocupan al hombre \\
\hline 7 & Provocación de la mujer \\
\hline
\end{tabular}

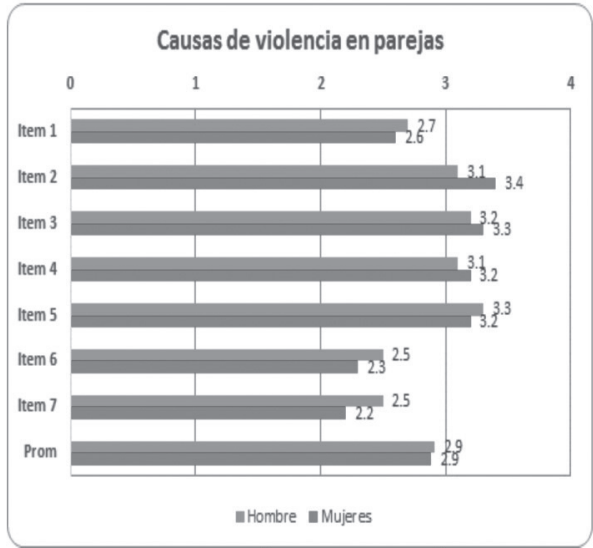


Ciertamente los estudiantes perciben varias causas de violencia en parejas. Con mínima diferencia, las mujeres indican estar de acuerdo con 3,4 puntos respecto a la existencia del "machismo o ideas machistas"; hombres y mujeres indican que hay trastornos mentales, abusos sufridos, consumo de drogas, alcohol como las causas que llevan a los agresores a violentar a la mujer. Cabe resaltar que, en el ítem 7, la tendencia es señalar que los estudiantes no están de acuerdo con que se deba a "provocación de la mujer", con 2,5.

\section{Valoraciones del amor y las relaciones de pareja en los estudiantes universitarios}

\section{Gráfico 3. Amor y relaciones de pareja}

\begin{tabular}{|c|l|}
\hline $\begin{array}{c}\text { İtem } \\
1\end{array}$ & $\begin{array}{l}\text { Amarse de verdad es necesario, pero no suficiente } \\
\text { para que una relación funcione. }\end{array}$ \\
\hline 2 & $\begin{array}{l}\text { El amor de verdad lo resiste todo, confiando en él se } \\
\text { superan todos los obstáculos. }\end{array}$ \\
\hline 3 & Amar a tu pareja y hacerle daño son incompatibles. \\
\hline 4 & $\begin{array}{l}\text { Como se suele decir, en las relaciones "quien bien te } \\
\text { quiere te hará sufrir". }\end{array}$ \\
\hline 5 & El amor lo perdona todo. \\
\hline 6 & No es cierto que haya que perdonar todo por amor. \\
\hline 7 & $\begin{array}{l}\text { En alguna parte hay alguien predestinado para cada } \\
\text { persona, su "media naranja". }\end{array}$ \\
\hline 8 & Lo de la media naranja es un cuento que tú no te crees. \\
\hline 9 & No existe un único amor verdadero. \\
\hline 10 & Solo se ama de verdad una vez en la vida. \\
\hline 11 & $\begin{array}{l}\text { Encontrar el amor significa encontrar a la persona que } \\
\text { dará sentido a tu vida. }\end{array}$ \\
\hline 12 & $\begin{array}{l}\text { El amor de pareja no es lo que da sentido a la vida de } \\
\text { una persona. }\end{array}$ \\
\hline 13 & $\begin{array}{l}\text { Por amor sería capaz de darlo todo sin esperar nada } \\
\text { a cambio. }\end{array}$ \\
\hline 14 & $\begin{array}{l}\text { Por amor sería capaz de implicarme y dar, pero a } \\
\text { cualquier precio. }\end{array}$ \\
\hline 15 & $\begin{array}{l}\text { Yo prefiero renunciar a la persona que amo antes que } \\
\text { dejar de ser yo mismo. }\end{array}$ \\
\hline 16 & $\begin{array}{l}\text { Yo cambiaría incluso algo que me gusta de mí para } \\
\text { conseguir a la persona que amo. }\end{array}$ \\
\hline 17 & Casarse o vivir juntos para siempre es la meta del amor \\
\hline 18 & Lo un cuento que tú no te crees. \\
\hline
\end{tabular}

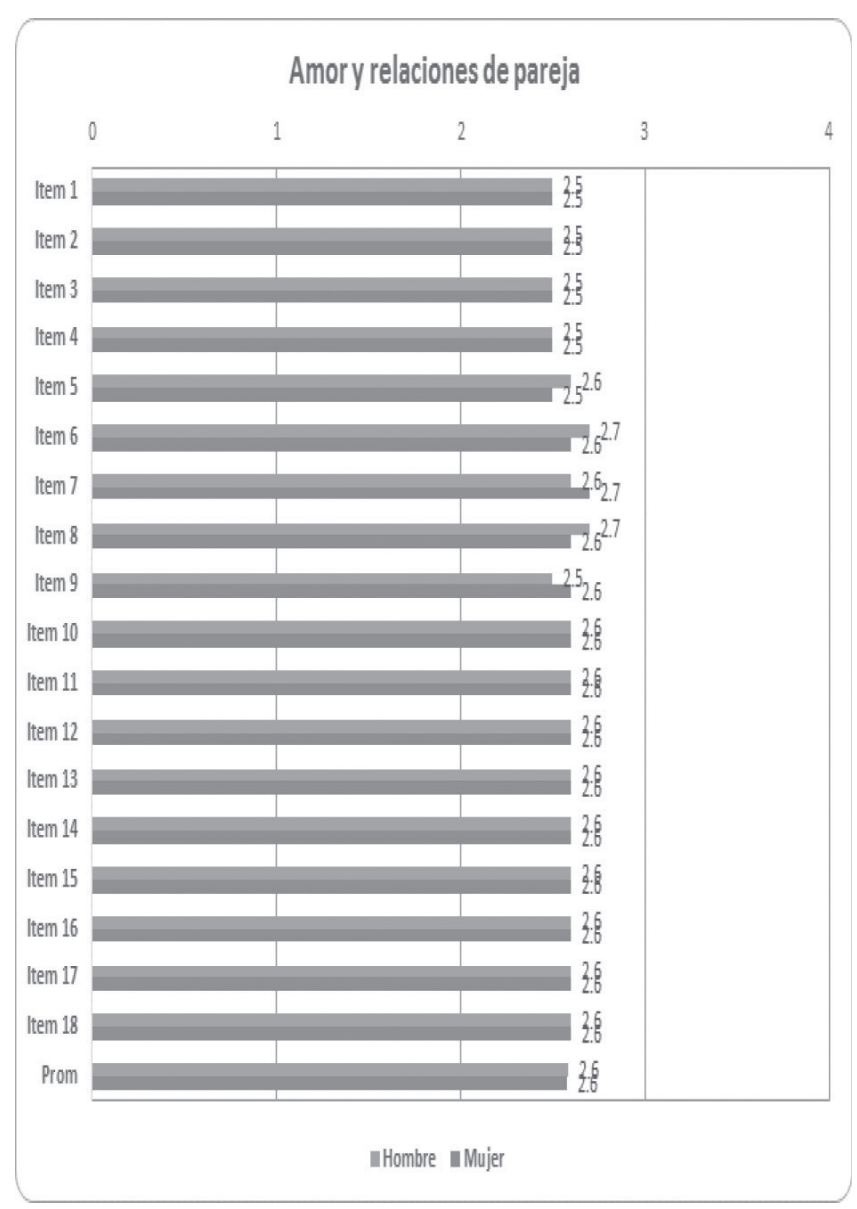


En general, todos los demás ítems son muy homogéneos y se orientan al "estar de acuerdo" con los ítems que se miden. Se puede analizar que las estudiantes están más conscientes de los riesgos de violencia y que hay sexismo; y en los hombres, indican que no harían esas acciones en relaciones de pareja. Pero los ítems 6, 7 y 8 muestran una relativa tendencia de "racionalidad" y de "expectativas" en las relaciones de pareja al considerar que hay "alguien que será tu media naranja"; pero que "no se puede perdonar todo por amor", es decir, hay una defensa de su autonomía. Es significativo que expresen que "por amor sería capaz de darlo todo sin esperar nada a cambio", en lo que están de acuerdo con 2,6 puntos. Están de acuerdo con que "casarse o vivir juntos para siempre es la meta del amor", diferenciándose de las percepciones y valoraciones de las estudiantes, es decir que coinciden sobre las valoraciones del amor de pareja por la cuales no es lógico que se dé violencia entre quienes se aman, lo que da consistencia a la discursiva encontrada en la percepción de los estudiantes.

\section{Gráfico 4. El amor significa encontrar a...}

\begin{tabular}{|c|l|}
\hline $\begin{array}{c}\text { Ítem } \\
1\end{array}$ & Alguien hecho a tu medida. \\
\hline 2 & La persona que dará sentido a tu vida. \\
\hline 3 & La persona que encajará contigo a la perfección. \\
\hline 4 & Alguien que te ame y te haga sentir bien contigo misma/o. \\
\hline 5 & La persona que te hará feliz para siempre. \\
\hline
\end{tabular}

Los estudiantes buscan una pareja que "Ie quiera y haga sentir bien mayor puntaje 3,2 en la valoración de los hombres, lo que estaría indicando que la afectividad es un área que priorizan como más estable los hombres.

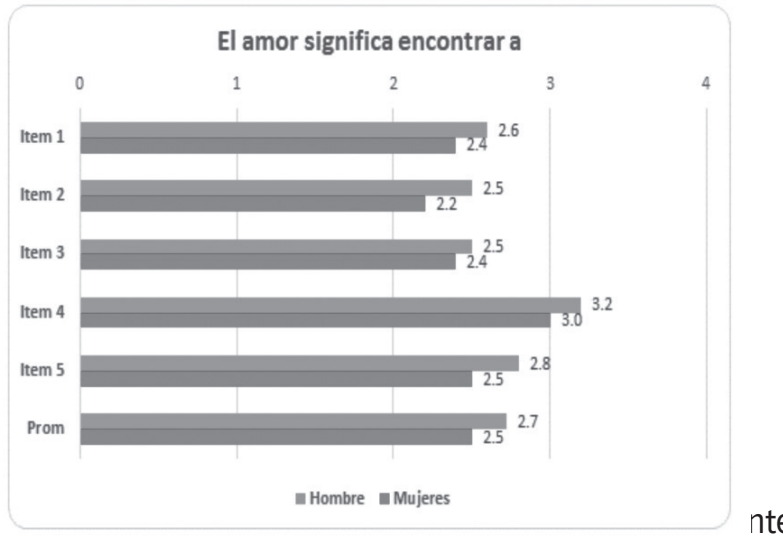

nte

Gráfico 5. Por amor sería capaz de...

\begin{tabular}{|c|l|}
\hline $\begin{array}{c}\text { Ítem } \\
1\end{array}$ & El amor es lo más importante en la vida. \\
\hline 2 & Entregarme, olvidándome de mí. \\
\hline 3 & Cambiar mi manera de vestir o mi estilo de vida. \\
\hline 4 & Implicarme y dar, pero no a cualquier precio. \\
\hline 5 & Darlo todo sin esperar nada a cambio. \\
\hline 6 & $\begin{array}{l}\text { Cambiar algo que me gusta de mí para conseguir a } \\
\text { quien amo. }\end{array}$ \\
\hline
\end{tabular}

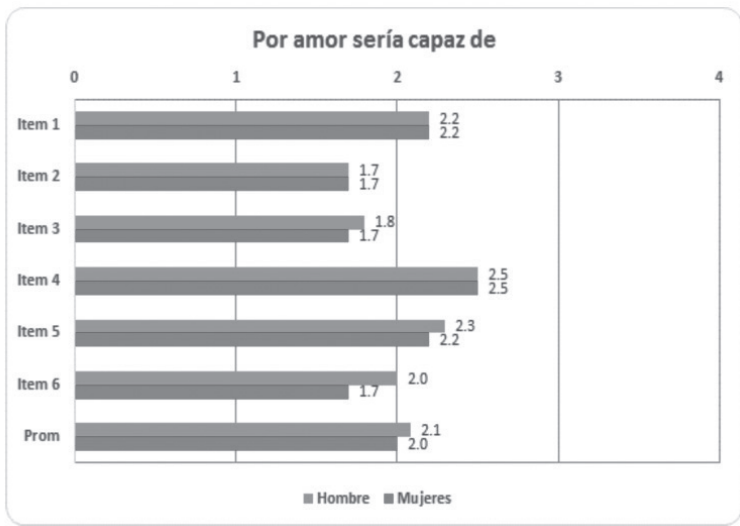


Los estudiantes expresan tener claro sus objetivos profesionales y un autoconcepto de sí mismos que priorizan; sin embargo, hay temor a los riesgos de violencia que puedan presentarse, por lo que indican "estar en desacuerdo" de hacer cosas por amor que puedan hacerles daño. La idea que "el amor es lo más importante en la vida" está más cerca a la valoración "en desacuerdo". Según los resultados, tenemos una constante en los cambios y valoraciones en las relaciones de pareja de los estudiantes, hombres y mujeres, universitarios.

\section{Gráfico 6. Si tu pareja te daña o hace sufrir}

\begin{tabular}{|c|l|}
\hline $\begin{array}{c}\text { Ítem } \\
1\end{array}$ & $\begin{array}{l}\text { Tiene solución si estás dispuesto/a perdonar lo que sea } \\
\text { y seguir adelante. }\end{array}$ \\
\hline 2 & Tiene solución si te pide perdón y te promete cambiar. \\
\hline 3 & $\begin{array}{l}\text { Tiene solución si lo ha hecho por amor, porque te quiere } \\
\text { demasiado. }\end{array}$ \\
\hline 4 & En esta situación lo mejor es cortar la relación. \\
\hline
\end{tabular}

Las estudiantes muestran "estar de acuerdo" que, frente al maltrato, es mejor terminar la relación, con cierta similitud con los estudiantes. En general, los estudiantes expresan

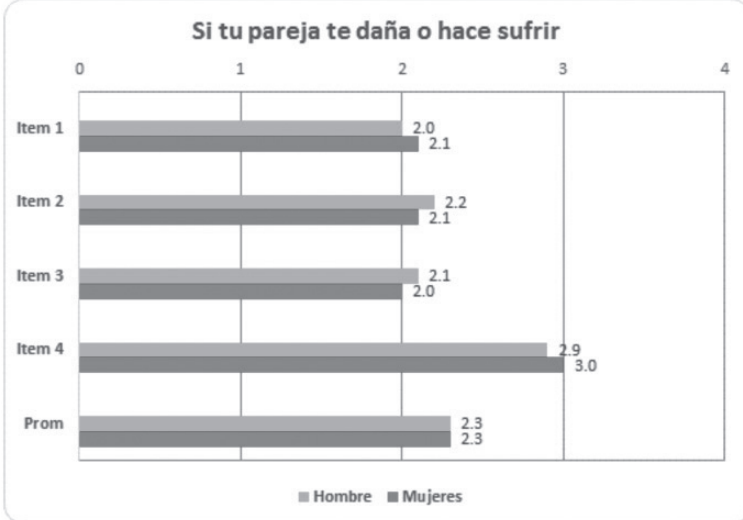

"estar en desacuerdo" en relaciones que afecten o creen violencia; pero están implicados en una relación a la cual le dan credibilidad y se comprometen.

\section{Las estrategias de afectividad en las relaciones de pareja en los estudiantes universitarios}

\section{Grafico 7. Percepción estrategias enamorados / noviazgo}

\begin{tabular}{|c|l|}
\hline $\begin{array}{c}\text { Ítem } \\
1\end{array}$ & Intimidar \\
\hline 2 & Agresión física \\
\hline 3 & Manifestar seísmo \\
\hline 4 & Abusos sexuales \\
\hline 5 & Desvalorizar \\
\hline 6 & Controlar \\
\hline 7 & Dominar \\
\hline 8 & Celos / posesión \\
\hline 9 & Aislar \\
\hline 10 & Chantaje emocional \\
\hline
\end{tabular}

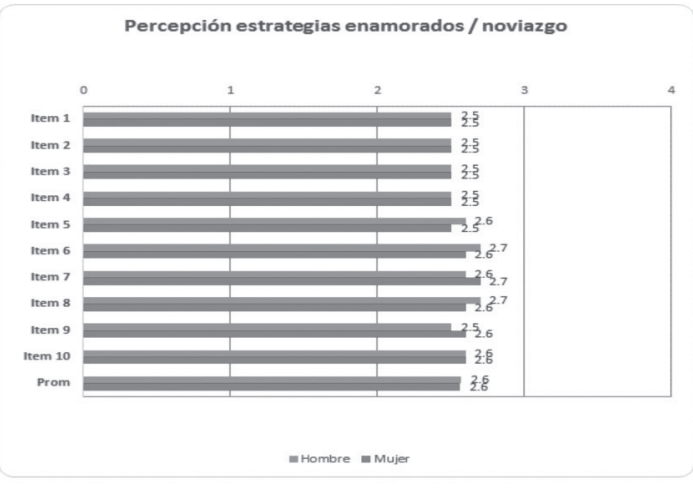


La percepción de los estudiantes es de "estar de acuerdo" que existen estrategias en las relaciones de pareja donde hay control, presión, subordinación, dominación/sujeción de la pareja; están en término medio, es decir, que puede ser sutil, pero se orienta al estar de acuerdo. Por tanto, se observa que hay un sexismo "benévolo". Destaca que los hombres indican que se sienten "controlados" por las mujeres; en tanto que las mujeres indican que se sienten "dominadas" por ellos.

\section{Gráfico 8. La frase que más te gustaría escuchar de tu pareja es}

\begin{tabular}{|c|l|}
\hline $\begin{array}{c}\text { İtem } \\
1\end{array}$ & Estás hecha/o para mí. \\
\hline 2 & Mi vida no tienen sentido sin ti. \\
\hline 3 & Soy solo tuya/o. \\
\hline 4 & Estar contigo es lo mejor que ha pasado nunca. \\
\hline 5 & Me encanta que sea solo mía/o. \\
\hline 6 & $\begin{array}{l}\text { Si tu pareja tiende a mostrar celos injustificados, } \\
\text { tienes un problema, estos celos son incompatibles } \\
\text { con el amor. }\end{array}$ \\
\hline 7 & $\begin{array}{l}\text { Si tu pareja tiende a mostrar celos injustificados, es } \\
\text { normal, los celos son una prueba de amor. }\end{array}$ \\
\hline
\end{tabular}

Emocionalmente las mujeres muestran orientarse al "estar de acuerdo" en esperar demostraciones explicitas de amor, como el "estas hecha para mí"; en tanto lo que los varones esperan es una frase como "estar contigo es lo mejor que ha pasado nunca", es decir, una orientación hacia el ego de la persona, si bien la diferencia no es distante en la percepción de las mujeres, lo que resalta es la asignación de roles género de masculinidad.

\section{Discusión}

Conocer los factores de riesgo asociados con la violencia y el sexismo en las relaciones de pareja hace necesario tener en cuenta el entorno sociocultural con el momento actual que viven. Hay relevancia en los resultados por las situaciones de violencia que suceden en el país, pero también por las creencias, actitudes sexistas y tolerantes que transmiten modelos diferentes de masculinidad y feminidad entre hombres y mujeres, modelos que representan las identidades estereotipadas y sexistas que refuerzan la autoridad y dominio del varón, así como de dependencia y necesidad de protección de la mujer; ello trasciende el campo personal,

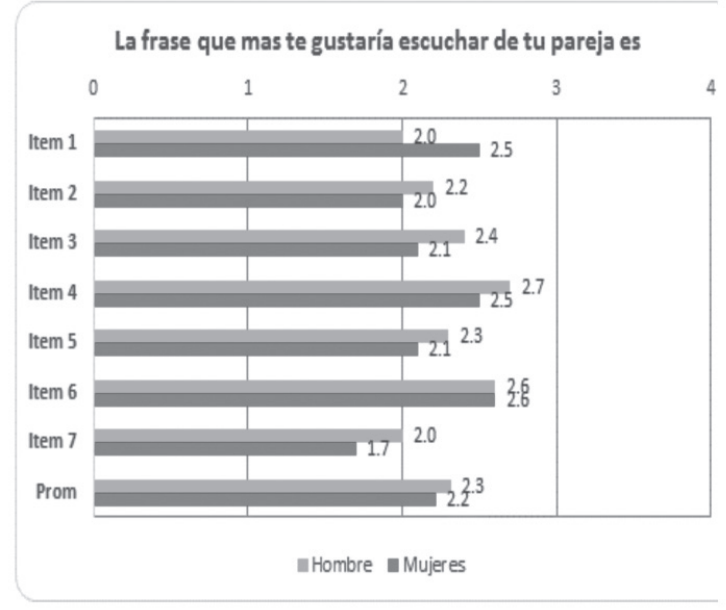

llegando a afectar las relaciones interpersonales en las que la violencia contra la mujer o las conductas sexuales se constituyen en riesgo.

Los estudios sobre sexismo benévolo paternalista, son las líneas centrales para comprender la asociación de la violencia con la afectividad de las relaciones de pareja caracterizadas por Rottenbacher (2010) sobre sexismo; hace comparaciones por áreas de estudio. Se tiene que existen diferencias significativas en el sexismo benévolo paternalista, lo que se acentúa en estudiantes de ingenierías con puntajes mayores, en comparación con los estudiantes de sociales. Esto puede ser un indicador de que en un ambiente donde las mujeres son minoría, como en las carreras de Ingeniería, podrían ser objeto de sexismo benévolo. Asimismo, tras hacer un análisis comparativo del sexismo en función de la universidad de procedencia, se encontró que los estudiantes de la universidad pública tenían mayores niveles de sexismo hostil, sexismo benévolo paternalista y sexismo ambivalente que quienes estudian en una universidad privada. Estos resultados pueden hacer hincapié en el hecho de que el sexismo está mediado por 
la condición socioeconómica de las personas. Es decir, que a menor estatus socioeconómico los papeles de género se vivencian de manera más tradicional y el sexismo se manifiesta de diversas maneras, tanto de manera hostil como benévola paternalista. Luna indica que en estratos socioeconómicos más bajos existe mayor sexismo y violencia en contra de la mujer (Luna, 2011), lo que nos lleva a relevar el factor social y educativo para eliminar las diversas formas de sexismo.

Respecto a las causas que crean violencia, hay estudios en los cuales se han evaluado las dificultades de salud física y mental de los victimarios de violencia hacia la pareja en la adolescencia o la juventud; han encontrado que las principales problemáticas de ese tipo son el uso o abuso de sustancias psicoactivas, la conducta sexual de riesgo y diferentes problemas de conducta externalizante (peleas, ser miembro de una pandilla, etc.).

Sin embargo, hay que distinguir, con los aportes de Castrillón (2001), los conceptos y performativa que tienen la violencia y la agresión, indicando que "agresión es la que se realiza sobre una víctima; la violencia es aquel estado de las relaciones sociales que para su mantenimiento o alteración precisa de una amenaza latente o explícita". Así, el concepto central de la violencia es el de amenaza, entendida como "la posibilidad de recibir daños de algún tipo no deseados por quien se opone a la premisa de mantenimiento o alteración"; afirma que la violencia no está determinada biológicamente, mientras que la agresividad sí; hace alusión a Domínguez (1998, p.46): “El agresivo nace y el violento se hace". (Castrillón, 2001).

En cambio, Bandura considera que las conductas pasan por procesos de modelamiento. Así, en la teoría del aprendizaje social (1978) propone que la conducta agresiva se adquiere bajo condiciones de modelamiento y por experiencias directas, y se define como una acción con características aversivas inherentes a ella, con cierto nivel de intensidad, que produce expresiones de ofensa o dolor y va acompañada de cierta intencionalidad, en donde juegan un papel importante las características de los agresores y de los mismos individuos que califican una conducta como agresiva. Se plantea una explicación para la agresión dentro de un proceso causal de dos vías, en el cual la conducta es controlable y el ambiente influenciable, por lo que el individuo crea un medio hostil, lo que a su vez crea individuos agresivos. La conducta agresiva, como toda clase de conducta, se activa mediante tres sistemas regulatorios, que son: a) motivo antecedente: control de estímulos, b) influencia de la retroalimentación de la respuesta: control de reforzamiento y c) procesos cognitivos: control cognitivo. Según lo anterior, se puede afirmar que las personas no nacen con repertorios preformados de conductas agresivas, sino que las adquieren, es decir, son aprendidas y controladas por cogniciones, contingencias de reforzamiento y por el ambiente (Ortiz, P.A.; Mindiola, C.; Mejía, W., 2001).

La teoría de socialización diferencial de género, que refiere al proceso de asignación de papeles o funciones y donde la sexualidad humana se aprende desde temprana edad; la afectividad y violencia en universitarios es estudiada, como es en el caso del amor y de las relaciones de pareja. Además, como señala Herrera (2011), las narraciones de los cuentos, as novelas, películas, canciones y otras producciones culturales influyen sobre nuestras expectativas y creencias mediante un sistema de "seducción" (muy ligado al consumo) que aumenta aún más la influencia y penetración de los mensajes que contienen (frente, por ejemplo, a la imposición o los imperativos presentes en otro tipo de mensajes). Así, mientras existe una correlación histórica y cultural entre masculinidad, violencia, agresividad y dominio, fomentando este tipo de comportamiento como prueba de virilidad, la socialización de las mujeres y las niñas incorpora elementos como la pasividad, la sumisión o la dependencia, que las hacen precisamente más vulnerables al padecimiento de comportamientos violentos y a la asunción del papel de víctimas (Herrera, 2011).

Otro enfoque permite comprender los resultados obtenidos en la presente investigación es el que señala Lorente, "que los cambios de papeles de las mujeres crea violencia". En el marco de estas nuevas formas de sexismo surgirían lo que Lorente (2009) ha denominado neomitos, es decir, nuevos mitos, evolucionados desde los tradicionales con el objeto de lanzar un nuevo mensaje, con un formato externo que parece rupturista con las posiciones tradicionales y que tiene la apariencia de neutralidad y defensa del bien común, pero que, en realidad, crea una referencia más compleja sobre las posiciones patriarcales tradicionales. Entre los ejemplos de estos nuevos mitos sobre la violencia de género estarían el llamado síndrome de alienación parental (SAP); la consideración de que las leyes criminalizan (considerando violencia de género) lo que son conflictos normales en las relaciones entre hombres y mujeres; la supuesta proliferación de denuncias falsas; o la consideración de los 
hombres como verdaderas víctimas del sistema (Lorente, 2009; Méndez, 2010).

Las posiciones más racionales de la percepción de los estudiantes en el estudio, de identificación de cambios en las valoraciones que tienen sobre su afectividad, se explican con estudios y análisis como los de Cobo (2011), quien precisa que "las mujeres se alejan cada vez más del estereotipo tradicional", y ello puede ser motivo de violencia del hombre, quien, en otras palabras, ve amenazado el orden de cosas que siempre fueron de su "dominio". Cobo (2011) indica que tanto la violencia individual como la colectiva, es decir, la ejercida por el hombre que golpea, insulta, humilla o asesina a su pareja al percibir como esta escapa a su control, y la ejercida por colectivos de hombres sobre el conjunto de las mujeres, para que, mediante el terror, sigan sometidas y se las impida llevar a cabo sus legítimas aspiraciones de autonomía personal y libertad de elección [como sería el caso del régimen talibán o de los feminicidios en Centroamérica] (Cobo, 2011). La violencia y su efecto en la afectividad de la mujer maltratada crean un mecanismo de "círculo vicioso" del cual es difícil salir para hallar una solución. Un vínculo humano fuerte e importante es el mayor factor resiliente para superar una experiencia de violencia (Oyague, 2003). Pero es justamente este vínculo el que es atacado por la violencia, entonces es difícil utilizarlo para recuperarse del trauma cuando es el vínculo fue arrebatado.

Todo lo expuesto permite sustentar, comparar y dar sentido a los resultados obtenidos. La problemática de violencia y sexismo en jóvenes y los códigos complejos del cortejo crean dependencia emocional que hace a las estudiantes más vulnerables. Por otro lado, hay necesidad de explicar y comprender a los estudiantes por cuanto también pasan por deterioro de salud mental, no siendo patologías en sí mismas, sino parte de un aprendizaje social —quizás hasta de forma inconsciente- de modelos de afectividad con su pareja que se normalizan por varios factores internos y externos que es necesario conocer; ello hace imprescindible dejar de poner a la mujer en papel de "victimización", pues debe haber o tenerse una visión proactiva y propositiva al respecto. Por cada marcha contra la no violencia, hay respuesta de mayor violencia, lo que indicaría que no es la única vía para aportar en la resolución de ésta problemática.

\section{Conclusiones}

1. En cuanto a las percepciones sobre la violencia sexual y el sexismo ambivalente, los resultados obtenidos ponen de manifiesto que el sexismo benevolente resulta el mejor predictor para la aceptación de mitos sobre la violencia sexual. Cabe resaltar que fue también esta dimensión del sexismo ambivalente la predominante en la muestra.

2. Existen cambios en las valoraciones y formas de mantener y construir la afectividad en las relaciones de pareja; por tanto, no es de esperarse que toda relación de pareja termine en violencia. Sin embargo, ello es vulnerable o de riesgo para las estudiantes, por cuanto la realidad ofrece ideas, acciones y valoraciones machistas, sexistas presentes en códigos de cortejo, galantería de los varones, lo que se expresan de forma sutil, en las relaciones de pareja y hace que las estudiantes no siempre puedan distinguir situaciones de riesgo. Así los estudiantes buscan una pareja que exprese que "le quiera y haga sentir bien consigo misma/o", lo es una forma de dependencia emocional.

3. Las causas de violencia y estrategias en las relaciones de pareja se sustentan en la vigencia del androcentrismo, y en un proceso de socialización diferencial de género que otorga jerarquías y valoraciones diferenciadas a la masculinidad y feminidad.

\section{Prospectiva y limitantes}

Esnecesaria lasocialización diferencial tempranaque respete la identidad según sexo biológico, pero que culturalmente las asignaciones sean de verdaderas oportunidades. Asimismo, frente a los resultados de la investigación y a escala nacional, es evidente que los hombres están creando y teniendo impactos desfavorables en su formación universitaria, es decir, requieren de ser comprendidos. Considero que hay que dar espacio a atender y focalizar a los estudiantes sobre sus concepciones de afectividad, ya sea en estudios o intervenciones profesionales. 


\section{Referencias}

Arias W. (2017) "La escala de sexismo ambivalente en estudiantes de dos Universidades Arequipa".

Bandura, A. Social learning theory. New Jersey: Prentice Hall; 1977.

Cárdenas, M.; Siu-lin, L.; González, C.; Calderón, C.; Alegría, I. (2010). "Inventario de sexismo ambivalente: adaptación, validación y relación con variables psicosociales". Revista Salud \& Sociedad V. 1; n. ${ }^{\circ}$ (pp. 125-135), ISSN 0718-7475. Universidad Católica del Norte, Chile.

Castrillón, M.; Vieco, G. (2002). "Actitudes justificativas del comportamiento agresivo y violento en estudiantes universitarios de la ciudad de Medellín. Colombia". Revista Facultad Nacional de Salud Pública (en línea), Julio-Diciembre. Consulta 27 de julio de 2018. Disponible en <http://www.redalyc.org/articulo. oa? id=12020205. ISSN 0120-386X

Cobo, R. (2011). Hacia una nueva política sexual. Las mujeres ante la reacción patriarcal. Madrid: Catarata.

De la Cruz, C. (2016). "Afectividad en mujeres víctimas y no víctimas de violencia de pareja atendidos en una clínica universitaria a través del psicodiagnóstico de Rorschach". PsiqueMag, Vol. 4, n. 1.

De la Peña, E.; Ramos, E.; Luzón, J.M.; Recio, P. (2011) Sexismo y Violencia en la juventud. Instituto Andaluz de la Mujer. Graficas Sabater. España.

Domínguez, R.; Revilla, J.C.; Gimeno, L. Jóvenes violentos: causas psicológicas de la violencia en grupo. Barcelona: Icaria, 1998. (p. 46).

Garaigordobil, M. (2015). “Sexismo y expresión de ira: Diferencias de género, cambios con la edad y correlaciones entre ambos constructos". Revista Argentina de Clínica Psicológica, 24(1) [pp. 35-42].

Herrera, C. (2011). La construcción sociocultural del amor romántico. Madrid: Fundamentos.

Luna, S.E. (2011). "Experiencia de masculinidad: la visión de un grupo de hombres guatemaltecos". Salud \& Sociedad, 2 (3) [pp. 250-266].

Lorente, M. (2009). Los nuevos hombres nuevos. Barcelona: Destino.

Méndez, R. (2010). "El SAP en su repercusión social". En A. Escudero, D. González, R. Méndez, C. Naredo, E. Pleguezuelos y S. Vaccaro, "Informe del Grupo de Trabajo de Investigación sobre el Supuesto síndrome de Alienación Parental" (pp. 76-94). Madrid: Ministerio de Igualdad.

Oyague, M. (2003). "Efectos de la violencia en la vida personal y familiar". Revista Páginas, 28 (pp. 46-56).

Ortiz, P.A.; Mindiola, C.; Mejía, W. (2001) "Estudio comparativo de las actitudes justificativas del comportamiento agresivo y violento en estudiantes de las universidades de Antioquia y Nacional (públicas) y la Cooperativa de Colombia (privada) de la ciudad de Medellín". Trabajo de grado (Psicología). Universidad de Antioquia. Facultad de Ciencias Sociales y Humanas. Medellín, 2001.

Rottenbacher, J.M. (2010). "Sexismo ambivalente, paternalismo masculino e ideología política en adultos jóvenes de la ciudad de Lima". Pensamiento Psicológico, 14 (pp.9-18).

Vara-Horna, A. \& López-Odar, D. (2016). "La violencia contra las mujeres en las universidades peruanas. Prevalencia e impacto en la productividad académica en las facultades de ciencias empresariales e ingenierías". Lima: GIZ \& USMP. 